\title{
A computational approach to linear conjugacy in a class of power law kinetic systems
}

\author{
Mark Jayson Cortez ${ }^{1}$ • Allen Nazareno ${ }^{1}$. \\ Eduardo Mendoza ${ }^{1,2,3,4}$
}

Received: 6 May 2017 / Accepted: 18 August 2017 / Published online: 30 August 2017

(C) The Author(s) 2017. This article is an open access publication

\begin{abstract}
This paper studies linear conjugacy of PL-RDK systems, which are kinetic systems with power law rate functions whose kinetic orders are identical for branching reactions, i.e. reactions with the same reactant complex. Mass action kinetics (MAK) systems are the best known examples of such systems with reactant-determined kinetic orders (RDK). We specify their kinetics with their rate vector and $T$ matrix. The $T$ matrix is formed from the kinetic order matrix by replacing the reactions with their reactant complexes as row indices (thus compressing identical rows of branching reactions of a reactant complex to one) and taking the transpose of the resulting matrix. The $T$ matrix is hence the kinetic analogue of the network's matrix of complexes $Y$ with the latter's columns of non-reactant complexes truncated away. For MAK systems, the $T$ matrix and the truncated $Y$ matrix are identical. We show that, on non-branching networks, a necessary condition for linear conjugacy of MAK systems and, more generally, of PL-FSK (power law factor span surjective kinetics) systems, i.e. those whose $T$ matrix columns are pairwise different, is $T=T^{\prime}$, i.e. equality of their $T$ matrices. This motivated our inclusion of the condition $T=T^{\prime}$ in exploring extension
\end{abstract}

Eduardo Mendoza
mendoza@1mu.de
Mark Jayson Cortez
mvcortez1@ up.edu.ph
Allen Nazareno
alnazareno@ up.edu.ph
$1 \quad \begin{aligned} & \text { Institute of Mathematical Sciences and Physics, University of the Philippines Los Baños, Laguna } \\ & \text { 4031, Philippines }\end{aligned}$
Institute of Mathematics, University of the Philippines Diliman, Quezon City 1101, Philippines
$3 \quad$ Max Planck Institute of Biochemistry, Martinsried, Munich, Germany
$4 \quad$ Faculty of Physics and Center for Nanoscience, Ludwig Maximilian University, Munich, Germany 
of results from MAK to PL-RDK systems. We extend the Johnston-Siegel Criterion for linear conjugacy from MAK to PL-RDK systems satisfying the additional assumption of $T=T^{\prime}$ and adapt the MILP algorithms of Johnston et al. and Szederkenyi to search for linear conjugates of such systems. We conclude by illustrating the results with several examples and an outlook on further research.

Keywords Chemical reaction networks · Dynamic equivalence $\cdot$ Linear conjugacy · Mixed integer linear programming · Power law kinetics · T matrix

\section{Abbreviations}

$\begin{array}{ll}\text { BST } & \text { Biochemical systems theory } \\ \text { CBK } & \text { Complex balanced kinetics } \\ \text { CFK } & \text { Comlplex factorizable kinetics } \\ \text { CKS } & \text { Chemical kinetic system } \\ \text { CRN } & \text { Chemical reaction network } \\ \text { CRNT } & \text { Chemical reaction network theory } \\ \text { JSC } & \text { Johnston-Seigel criterion } \\ \text { GMAK } & \text { Generalized mass action kinetics } \\ \text { KSS } & \text { Kinetic and stoichiometric subspaces } \\ \text { KSSC } & \text { KSS coincidence } \\ \text { MAK } & \text { Mass action kinetics } \\ \text { ODE } & \text { Ordinary differential equations } \\ \text { PLK } & \text { Power-law kinetics } \\ \text { PL-FSK } & \text { Power-law factor span surjective kinetics } \\ \text { PL-NDK } & \text { Power-law non-reactant-determined kinetics } \\ \text { PL-RDK } & \text { Power-law reactant-determined kinetics } \\ \text { PT } & \text { Point-terminal } \\ \text { RDK } & \text { Reactant-determined kinetic orders } \\ \text { SCC } & \text { Stoichiometric compatibility class } \\ \text { SFRF } & \text { Species formation rate function }\end{array}$

\section{List of symbols}

$\begin{array}{ll}\delta & \text { Deficiency of a CRN } \\ \psi_{K} & \text { Factor map of a complex factorizable kinetics } K \\ I_{a} & \text { Incidence map of a CRN } \\ \delta_{k} & k \text {-deficiency } \\ I_{k} & k \text {-incidence map } \\ A_{k} & k \text {-Laplacian map } \\ K & \text { Kinetic subspace of a CKS } \\ Y & \text { Molecularity map/ matrix of complexes } \\ \mathscr{K}(\mathscr{N}) & \text { Set of all chemical kinetics on a CRN } \mathscr{N} \\ f & \text { Species formation rate function of a CKS } \\ S & \text { Stoichiometric subspace of a CKS } \\ t-l & \text { Terminality }\end{array}$




\section{Introduction}

Researchers studying dynamical systems based on chemical reaction networks with mass action kinetics (MAK) were aware from the beginning that different networks could generate the same set of ordinary differential equations (ODE), i.e. be dynamically equivalent. An important step in the systematic study of dynamical equivalence was taken by G. Farkas [6], who investigated kinetic lumping schemes for MAK systems i.e. exact linear lumping schemes, which preserve the structure of the original kinetic system. The mathematical foundations of dynamical equivalence were established nine years later in the paper of G. Craciun and C. Pantea [4] and the commentary note of G. Szederkenyi [18]. Two years later, M. Johnston and D. Siegel extended the studies to linear conjugacy of MAK systems and formulated a criterion for the property [12].

In the past five years, various authors have pioneered the use of mixed integer linear programming (MILP) algorithms for determining linear conjugacy between mass action kinetics (MAK) systems. In particular, the special case of dynamical equivalence-the classical question of model identifiability-has received a lot of attention. A good review of the work until 2013 in Realization Theory-as the field is sometimes called by CRNT researchers-is provided in Johnston et al [14]. More recently, Johnston [11] extended the algorithm described there to find linear conjugates satisfying the Deficiency One Theorem for MAK systems.

To our knowledge, only two papers have been so far published, which use MILP approaches for linear conjugacy and dynamical equivalence of systems with non-MAK kinetics. In the first paper [7], Gabor et al. addressed systems with kinetics which are rational functions (e.g. Michaelis-Menten and Hill type systems) by extending the canonical method of Hars and Toth for representing MAK systems as CRNs [9] and the algorithms of Szederkenyi [19] for dense and sparse realizations to such rational function systems. In the second paper, Johnston [10] applied MILP algorithms on translation of MAK to Generalized Mass Action Kinetics (GMAK) systems as defined by Müller and Regensburger in [15], which we denote by GMAK-12 systems. This translation process transforms toric equilibria of weakly reversible MAK systems to complex balanced equilibria of weakly reversible GMAK-12 systems with zero kinetic deficiency.

In this paper, we extend the Johnston-Siegel Criterion (JSC) for Linear Conjugacy [12] from MAK systems to PL-RDK (Power Law with Reactant-Determined Kinetic orders) and adapt the JSC-based MILP algorithm of Johnston et al. [14] to the latter. PL-RDK systems are the power law kinetic systems for which for which the rows in the kinetic order matrix are identical if the reactions have the same reactant complex, i.e. are branching reactions of the same reactant complex. Formally, if the power law system on a network $(\mathscr{S}, \mathscr{C}, \mathscr{R})$ is given by $\frac{d x}{d t}=N \operatorname{diag}(k) x^{F}$, where $N$ is its stoichiometric matrix, $k$ the rate vector, $F$ the kinetic order matrix and $\rho: \mathscr{R} \rightarrow \mathscr{C}$ is the reactant map, the system is PL-RDK if $\rho(r)=\rho\left(r^{\prime}\right)$ implies $F_{r, .}=F_{r^{\prime}, .}$. In [1], it is shown that PL-RDK systems are precisely the power law systems for which a factorization $\frac{d x}{d t}=Y A_{k} \Psi_{K}$ exists, where $Y$ is the matrix of complexes, 
$A_{k}$ the Kirchhoff matrix and the factor map $\Psi_{K}=x^{F}$. Clearly, MAK systems form a subset of PL-RDK. The correspondence between PL-RDK systems and the Generalized Mass Action Kinetics introduced by Müller and Regensburger in [16] (which we call GMAK-14) are discussed in detail in [21].

We specify the kinetics of PL-RDK systems with their rate vector and $T$ matrix. The $T$ matrix is formed from the kinetic order matrix by replacing the reactions with their reactant complexes as row indices (thus compressing identical rows of branching reactions of a reactant complex to one) and taking the transpose of the resulting matrix. The $T$ matrix is hence the kinetic analogue of the network's matrix of complexes $Y$ with the latter's columns of non-reactant complexes truncated away. For MAK systems, the $T$ matrix and the truncated $Y$ matrix are identical.

The set PL-FSK (Power Law Factor Span Surjective Kinetics) is an important subset of PL-RDK and consists of systems with the property that reactions with different reactant complexes have different kinetic order matrix rows. In other words, $\rho(r) \neq$ $\rho\left(r^{\prime}\right)$ implies $F_{r, .} \neq F_{r^{\prime}, .}$. As shown in [1], this property is equivalent to the linear independence of the non-zero coordinate functions of the factor map $\Psi_{K}$. Again, MAK systems form a subset of PL-FSK, and various properties of MAK systems can be extended to PL-FSK, e.g. the Feinberg-Horn Theorem on the coincidences of the kinetic and stoichiometric subspaces [1]. The GMAK-14 systems whose map of kinetic complexes $\tilde{y}: \rho(\mathscr{R}) \rightarrow \mathbb{R}^{\mathscr{S}}$ is injective correspond to PL-FSK. A property of PL-FSK systems on linearly conjugate nonbranching networks $N$ and $N^{\prime}$ motivated the inclusion of the condition $T=T^{\prime}$ in our extension of the MAK results (s. Sect. 3).

\section{Basic concepts and notation}

In this section, we collect the concepts and propositions of CRNT that we need to derive our results.

Definition 1 A chemical reaction network $(\mathrm{CRN}) \mathscr{N}$ is a triple $(\mathscr{S}, \mathscr{C}, \mathscr{R})$ of finite sets of species in $\mathscr{S}$, complexes in $\mathscr{C} \subset \mathbb{R}^{\mathscr{S}}$, and reactions in $\mathscr{R} \subset \mathscr{C} \times \mathscr{C}$ such that

1. $(y, y) \notin \mathscr{R}$ for any $y \in \mathscr{C}$;

2. for each $y \in \mathscr{C}$ there exists $y^{\prime} \in \mathscr{C}$ such that $\left(y, y^{\prime}\right) \in \mathscr{R}$ or $\left(y^{\prime}, y\right) \in \mathscr{R}$.

For a CRN $\mathscr{N}=(\mathscr{S}, \mathscr{C}, \mathscr{R})$, we denote with $m$ the number of species in $\mathscr{S}, n$ the number of complexes in $\mathscr{C}$, and $r$ the number of reactions in $\mathscr{R}$.

The pair $(\mathscr{C}, \mathscr{R})$ defines a digraph called the "reaction graph" of the $\mathrm{CRN}$ and is often used to describe the network. However, the reaction graph determines the triple uniquely only if an additional property is included in the definition: $\mathscr{S}=$ $\cup\{$ supp $y \mid y \in \mathscr{C}\}$, i.e. each species appears in a complex. CRNs with this additional property can equivalently be defined as:

Definition 2 A chemical reaction network $(\mathrm{CRN})$ is a digraph $(\mathscr{S}, \mathscr{C}, \mathscr{R})$ where each vertex has positive degree and stoichiometry, i.e. there is a finite set $\mathscr{S}$ (whose elements are called species) such that $\mathscr{C}$ is a subset of $\mathbb{R}_{\geq}^{\mathscr{S}}$. Each vertex is called a complex and its coordinates in $\mathbb{R}_{\geq}^{\mathscr{S}}$ are called stoichiometric coefficients. The arcs are called reactions. 
The connectivity concepts in digraph theory apply to CRNs, but have slightly differing names since a connected component is traditionally called a linkage class in CRNT. Hence, a strong linkage class is subset of a linkage class where any two elements are connected by a directed path in each direction. A terminal strong linkage class is a strong linkage class such that there is no reaction from a complex in the strong linkage class to a complex not in the strong linkage class.

We now introduce the set of power law kinetics and the subsets that we will focus on.

Definition 3 A kinetics for a network $\mathscr{N}=(\mathscr{S}, \mathscr{C}, \mathscr{R})$ is an assignment to each reaction $r_{j} \in \mathscr{R}$ of a rate function $K_{j}: \Omega_{K} \rightarrow \mathbb{R}_{\geq}$, where $\Omega_{K}$ is a set such that $\mathbb{R}_{>} \subseteq \Omega_{K} \subseteq \mathbb{R}_{\geq}, c \wedge d \in \Omega_{K}$ whenever $c, d \in \Omega_{K}$, and $K_{j}(c) \geq 0, \forall c \in \Omega_{K}$.

A kinetics for a network $\mathscr{N}$ is denoted by $K=\left(K_{1}, K_{2}, \ldots, K_{r}\right): \Omega_{K} \rightarrow \mathbb{R}_{\geq}^{\mathscr{R}}$. In the definition, $c \wedge d$ is the bivector of $c$ and $d$ in the exterior algebra of $\mathbb{R}^{\mathscr{S}}$. Our work is focused on the following subset:

Definition 4 A chemical kinetics is a kinetics $K$ satisfying the positivity condition: for each reaction $r_{j}: y \rightarrow y^{\prime}, K_{j}(c)>0$ iff supp $y \subset \operatorname{supp} c$.

If $\Omega_{K}=\mathbb{R}_{>}^{\mathscr{S}}$, then the additional condition for a chemical kinetics simply says that $K_{j}(c)>0$ for all $c$, hence the name "positivity condition".

Example 1 Let $\Omega_{K}=\mathbb{R}_{>}^{\mathscr{S}}$ and $F$ an $r \times m$ matrix of real numbers. Define $x^{F}$ by $\left(x^{F}\right)_{i}=\prod x_{j}^{F_{i j}}$ for $i=1, \ldots, r$. The kinetics defined by $k_{i} x_{i}^{F}$ is called a power law kinetics (PLK) and the matrix $F$ the kinetic order matrix.

Mass action kinetics is clearly a special case of this kinetics set. We are particularly interested in two MAK $(\mathscr{N})$ supersets with similar properties. The first is the set with reactant-determined kinetic orders $\mathscr{P} \mathscr{L}-\mathscr{R} \mathscr{D} \mathscr{K}(\mathscr{N})$.

Definition 5 A PLK system has a reactant-determined kinetic orders (of type PLRDK) if for any two reactions $i, j$ with identical reactant complexes, the corresponding rows of kinetic orders in $V$ are identical, i.e., $v_{i k}=v_{j k}$ for $k=1,2, \ldots, m$.

$\mathscr{P} \mathscr{L}-\mathscr{R} \mathscr{D} \mathscr{K}(\mathscr{N})$ includes mass action kinetics (MAK) and coincides with the set of GMAK-14 systems recently introduced by Müller and Regensburger [16]. They also constitute the subset of power law systems for which the claim of various authors that their results "hold for complexes with real coefficients" are valid.

The superset of MAK systems of highest interest for us is the set of factor span surjective systems $\mathscr{P} \mathscr{L}-\mathscr{F} \mathscr{S} \mathscr{K}(\mathscr{N})$. We use the following characterization which was derived in [1] as our working definition:

Definition 6 A PL-RDK kinetics is factor span surjective if and only if all rows with different reactant complexes in the kinetic order matrix $F$ are pairwise different (i.e. $\rho(r) \neq \rho\left(r^{\prime}\right)$ implies $\left.F_{r, .} \neq F_{r^{\prime}, .}\right)$.

If the given kinetics is of type PL-RDK, we can define the factor map of the Chemical Kinetic System (CKS). The definition below came from Arceo et al. [1]. 
Definition 7 The factor map $\psi_{K}: \mathbb{R}^{m} \rightarrow \mathbb{R}^{n}$ is defined as

$$
\left(\psi_{K}\right)_{c}(x)= \begin{cases}\left(x^{F}\right)_{i}, & \text { if } c \text { is a reactant complex of a reaction } i \\ 0, & \text { otherwise }\end{cases}
$$

A further important concept for a PL-RDK system is the Kirchhoff matrix of the system:

Definition 8 The Kirchhoff matrix (also called the kinetics matrix or Laplacian matrix) of a PL-RDK system is the $m \times n$ matrix defined by

$$
\left(A_{k}\right)_{i j}= \begin{cases}k_{j i}, & \text { if } i \neq j \\ k_{j j}-\sum_{x=1}^{n} k_{j x}, & \text { if } i=j\end{cases}
$$

where $k_{j i}$ is the rate constant associated to the reaction from $C_{i}$ to $C_{j}$.

The SFRF $f$ has the following decomposition: $f(x)=Y \circ A_{k} \circ \psi_{K}(x)$, indicating as shown in [1] that, for any network $\mathscr{N}$,

$\mathscr{P} \mathscr{L}-\mathscr{R} \mathscr{D} \mathscr{K}(\mathscr{N})=\mathscr{P} \mathscr{L} \mathscr{K}(\mathscr{N}) \cap \mathscr{C} \mathscr{F} \mathscr{K}(\mathscr{N})$, where $\mathscr{C} \mathscr{F} \mathscr{K}(\mathscr{N})$ is the set of complex factorizable kinetics on $\mathscr{N}$.

$\mathscr{P} \mathscr{L}-\mathscr{F} \mathscr{S} \mathscr{K}(\mathscr{N})$ is a large and important subset of $\mathscr{P} \mathscr{L}-\mathscr{R} \mathscr{D} \mathscr{K}(\mathscr{N})$ which reflects important properties of its subset of MAK systems. For example, as shown in [1], the Feinberg-Horn Theorem on the coincidence of kinetic and stoichiometric subspaces extends precisely to this set of power law kinetics.

We recall the definition of the $m \times n$ matrix $\tilde{Y}$ from [16]: for a reactant complex, the column of $\tilde{Y}$ is the transpose of the kinetic order matrix row of the complex's reaction, otherwise (i.e. for a terminal point), the column is 0 .

Definition 9 The $T$ matrix of a PL-RDK system is formed by truncating away the columns of the terminal points in $\tilde{Y}$, obtaining an $m \times n_{r}$ matrix. The corresponding linear map $T: \mathbb{R}^{\rho(\mathscr{R})} \rightarrow \mathbb{R}^{\mathscr{S}}$ maps $\omega_{\rho(r)}$ to $\left(F_{r}\right)^{T}$. The subspace $\tilde{R}:=\operatorname{Im} T=$ $\left\langle\left(F_{r}\right)^{T}\right\rangle$ is called the kinetic reactant subspace and $\tilde{q}=\operatorname{dim} \tilde{R}$ is called the kinetic reactant rank of the system.

Remark 1 Müller and Regensburger introduced the map (of sets) $\tilde{y}: \rho(\mathscr{R}) \rightarrow \mathbb{R}^{\mathscr{S}}$ and called an element of its image a kinetic complex. Clearly $T\left(\omega_{y}\right)=\tilde{y}(y)$ for any reactant complex. If we denote the number of kinetic complexes with $\tilde{n}_{r}$, then we have $\tilde{q} \leq \tilde{n}_{r} \leq n_{r}$.

For the background on Biochemical Systems Theory (BST) and the CRN representations (total and embedded) of BST models needed for Examples 5 and 6, the reader is referred to the discussion in [1].

Two kinetic systems $M$ and $M^{\prime}$, though differing in structure, may give rise to a similar dynamics. This property, called linear conjugacy, is characterized by trajectories which are related by a linear transformation. If this mapping is trivial, $M$ and $M^{\prime}$ are said to be dynamically equivalent. 
Definition 10 Let $\left(\Phi\left(x_{0}, t\right)\right.$ and $\left(\Psi\left(x_{0}, t\right)\right.$ be flows associated to kinetic systems $M$ and $M^{\prime}$ respectively. $M$ and $M^{\prime}$ are said to be linearly conjugate if there exists a bijective linear mapping $h: \mathbb{R}_{>0}^{n} \rightarrow \mathbb{R}_{>0}^{n}$ such that $h\left(\Phi\left(x_{0}, t\right)\right)=\Psi\left(h\left(x_{0}, t\right)\right)$ for all $x_{0} \in \mathbb{R}_{>0}^{n}$.

\section{A necessary condition for linear conjugacy in PL-FSK systems}

In this section, we briefly describe our motivation to include the assumption $T=T^{\prime}$ in the extension of the MAK results on linear conjugacy to PL-RDK systems. We first observed that for some MAK systems with same sets of species and complexes, linear conjugacy implied that their sets of reactants coincided too. Further investigations then showed that this necessary condition was true for all PL-FSK systems on linearly conjugate nonbranching networks, as shown in the following proposition.

Proposition 1 Let $\mathscr{N}=(\mathscr{S}, \mathscr{C}, \mathscr{R})$ and $\mathscr{N}^{\prime}=\left(\mathscr{S}^{\prime}, \mathscr{C}^{\prime}, \mathscr{R}^{\prime}\right)$ be nonbranching networks and $(\mathscr{N}, K)$ and $\left(\tilde{\mathscr{N}}, K^{\prime}\right)$ be PL-FSK systems and linearly conjugate. Then $T=T^{\prime}$.

Proof Since $\mathscr{N}$ is nonbranching, $n_{r}=r$. Furthermore, $K$ is factor span surjective if and only if $\psi_{K, 1}, \ldots, \psi_{K, r}$ are linearly independent, where $\psi_{K, i}(x)=\prod_{j} x_{j}^{T_{j i}}$. For convenience, we write $P_{K}(i)=T ., i$ (the i-th column of $T$ ) and consider $P_{K}$ as a map $\mathscr{R} \rightarrow R^{\mathscr{S}}$. According to Proposition 14 of [1], this linear independence is equivalent to $P_{K}(i) \neq P_{K}(j)$ if $i \neq j$. Hence each network has a decomposition into $r$ and $r^{\prime}$ single reaction subnetworks respectively, which we refer to as their CF-decompositions. On the other hand, linear conjugacy implies for the SFRFs that $f-C f^{\prime}=0$. Expressing both SFRFs in their CF-decomposition results in a linear dependency relation between the constituent monomials. The summands of the LHS can be separated into the set of monomials only in $f$ and not in $f^{\prime}$, those common to both and the set of those in $f^{\prime}$ but not in $f$. A monomial in the first set is pairwise different from those in the union of the first and second set (except itself) since $K$ is factor span surjective and $\mathscr{N}$ nonbranching. By definition, this is also the case with those in the third set. The same argument applies to the third set by symmetry. Hence, the monomials on the LHS are linearly independent. This implies that the coefficients for the monomials in the first and third sets are all zero, i.e. both sets are empty. The remaining monomials are bijectively mapped to $\operatorname{Im} P_{K}=\operatorname{Im} P_{K}^{\prime}$. This implies $r=r^{\prime}$ and is equivalent to $T=T^{\prime}$ after appropriate ordering of the columns.

Since all MAK systems are factor span surjective, we have the following corollary:

Corollary 1 If $\mathscr{N}=(\mathscr{S}, \mathscr{C}, \mathscr{R})$ and $\mathscr{N}^{\prime}=\left(\mathscr{S}^{\prime}, \mathscr{C}^{\prime}, \mathscr{R}^{\prime}\right)$ are nonbranching and linearly conjugate, then $\rho(\mathscr{R})=\rho\left(\mathscr{R}^{\prime}\right)$.

Since for at least two significant sets of PL-RDK kinetics, $T=T^{\prime}$ is a necessary condition for linear conjugacy, we add this property to extend the Johnston-Siegel Theorem from MAK to PL-RDK systems in the next section. 


\section{The MILP algorithm for linear conjugacy in PL-RDK systems}

In this section, we present an MILP algorithm to construct PL-RDK systems, whose underlying networks are weakly reversible, and are linearly conjugate to a given system. We first extend the JSC for linear conjugacy for MAK systems to PL-RDK systems with the additional property that $T=T^{\prime}$. This serves as the basis for the adaptation of the MILP algorithm of Johnston et al. [13] for MAK systems to these PL-RDK systems. We conclude with several examples illustrating the extended MILP algorithm.

\subsection{Extension of the Johnston-Siegel Criterion to PL-RDK systems}

We restate the Johnston-Siegel Criterion for MAK systems as a reference for the discussion in this and the following sections.

Theorem 1 [12] Consider two mass-action systems $\mathscr{N}=(\mathscr{S}, \mathscr{C}, \mathscr{R})$ and $\mathscr{N}^{\prime}=$ $\left(\mathscr{S}^{\prime}, \mathscr{C}^{\prime}, \mathscr{R}^{\prime}\right)$ and let $Y$ be the stoichiometric matrix corresponding to the complexes in either network. Consider a kinetics matrix $A_{k}$ corresponding to $\mathscr{N}$ and suppose that there is a kinetics matrix $A_{b}$ with the same structure as $\mathscr{N}^{\prime}$ and a vector $c \in \mathbb{R}_{>0}^{n}$ such that

$$
Y \cdot A_{k}=C \cdot Y \cdot A_{b}
$$

where $C=\operatorname{diag}\{c\}$. Then $\mathscr{N}$ is linearly conjugate to $\mathscr{N}^{\prime}$ with kinetics matrix

$$
A_{k}^{\prime}=A_{b} \cdot \operatorname{diag}\{\psi(c)\} \text {. }
$$

To extend the criterion for linear conjugacy, we first show that the hypothesis $T=T^{\prime}$ ensures the equality of the factor maps of the pair of kinetics:

Proposition 2 Consider two PL-RDK systems $\mathscr{N}$ and $\mathscr{N}^{\prime}$. Then $T=T^{\prime}$ if and only if $\psi_{K}=\psi_{K}^{\prime}$.

Proof $(\Rightarrow)$ If $T=T^{\prime}$ then $\forall i, j T_{i j}=T_{i j}^{\prime}$. If complex $j$ is not a reactant complex, it corresponds to no column in $T$ and $T^{\prime}$. Hence, $\psi_{K_{j}}=\psi_{K_{j}}^{\prime}=0$. For any complex $j$ for which species $i$ is involved and reaction $k$ for which complex $j$ is a reactant, $F_{k i}=F_{k i}^{\prime}$ implies $\psi_{K_{j}}=\psi_{K_{j}}^{\prime}$.

$(\Leftarrow)$ If $\psi_{K}=\psi_{K}^{\prime}$ then for each reactant complex $j, \psi_{K_{j}}=\psi_{K_{j}^{\prime}}^{\prime}$ and $x_{j}^{F}=x_{j}^{F^{\prime}}$. Since for each reaction $k$ for which complex $j$ is a reactant $F_{k i}=F_{k i}^{\prime}$, then $T=T^{\prime}$.

We can now prove the extension of the JSC for PL-RDK systems:

Theorem 2 Consider two PL-RDK systems $\mathscr{N}=(\mathscr{S}, \mathscr{C}, \mathscr{R})$ and $\mathscr{N}^{\prime}=\left(\mathscr{S}^{\prime}\right.$, $\left.\mathscr{C}^{\prime}, \mathscr{R}^{\prime}\right)$ and let $Y$ be the matrix of complexes in either network. Suppose further that $T=T^{\prime}$. Consider a kinetics matrix $A_{k}$ corresponding to $\mathscr{N}$ and suppose that there is a kinetics matrix $A_{b}$ with the same structure as $\mathscr{N}^{\prime}$ and a vector $c \in \mathbb{R}_{>0}^{n}$ such that

$$
Y \cdot A_{k}=C \cdot Y \cdot A_{b}
$$


where $C=\operatorname{diag}\{c\}$. Then $\mathscr{N}$ is linearly conjugate to $\mathscr{N}^{\prime}$ with kinetics matrix

$$
A_{k}^{\prime}=A_{b} \cdot \operatorname{diag}\left\{\psi_{K}(c)\right\} .
$$

Proof Let $\varphi\left(x_{o}, t\right)$ be the solution of the system of ODE $\dot{x}=f(x)=Y A_{k} \psi_{K}$ associated to the reaction network $\mathscr{N}$.

Consider the linear map $h(x)=C^{-1} x$ where $C=\operatorname{diag}\{c\}, c \in \mathbb{R}_{>0}^{n}$.

Let $\tilde{\varphi}\left(y_{0}, t\right)=C^{-1} \varphi\left(x_{0}, t\right)$ so that $\varphi\left(x_{0}, t\right)=C \tilde{\varphi}\left(y_{0}, t\right)$. It follows that

$$
\begin{aligned}
\tilde{\varphi}^{\prime}\left(y_{0}, t\right) & =C^{-1} \varphi^{\prime}\left(x_{0}, t\right) \\
& =C^{-1} Y A_{k} \psi_{K}\left(\varphi\left(x_{0}, t\right)\right) \\
& =C^{-1} C Y A_{b} \psi_{K}\left(C \tilde{\varphi}\left(y_{0}, t\right)\right)
\end{aligned}
$$

Now,

$$
\begin{aligned}
\psi_{K}\left(C \tilde{\varphi}\left(y_{0}, t\right)\right) & =\psi_{K}\left(\operatorname{diag}\{c\} \tilde{\varphi}\left(y_{0}, t\right)\right) \\
& =D \psi_{K}\left(C \tilde{\varphi}\left(y_{0}, t\right)\right)
\end{aligned}
$$

where $D=\operatorname{diag}\{e\}$ and $e_{j}= \begin{cases}c^{F \cdot j}, & \text { if complex } j \text { is a reactant of some reaction } k \\ 1, & \text { otherwise }\end{cases}$ So, $\tilde{\varphi}^{\prime}\left(y_{0}, t\right)=Y A_{b} D \psi_{K}\left(\tilde{\varphi}\left(y_{0}, t\right)\right)$. Clearly, $\tilde{\varphi}\left(y_{0}, t\right)$ is a solution of the system $\dot{x}=Y A_{b} D \psi_{K}$ corresponding to the reaction network $\mathscr{N}^{\prime}$. We have that $h\left(\varphi\left(x_{0}, t\right)\right)=\tilde{\varphi}\left(h\left(x_{0}\right), t\right)$ for all $x_{0} \in \mathbb{R}_{>0}^{n}$ and $t \geq 0$ where $y_{0}=h\left(x_{0}\right)$ since $y_{0}=\tilde{\varphi}\left(y_{0}, t\right)=C^{-1} y_{0}=\varphi\left(y_{0}, t\right)$. It follows that networks $\mathscr{N}$ and $\mathscr{N}^{\prime}$ are linearly conjugate.

Remark 2 Again, we see from the proof that, if $\mathscr{S}=\mathscr{S}^{\prime}$, for the linearly conjugate system constructed, $\mathscr{C}=\mathscr{C}^{\prime}$. We can also show that the set of reactant complexes coincide:

Proposition 3 Consider PL-RDK systems $\mathscr{N}=(\mathscr{S}, \mathscr{C}, \mathscr{R})$ and $\mathscr{N}^{\prime}=\left(\mathscr{S}^{\prime}, \mathscr{C}^{\prime}, \mathscr{R}^{\prime}\right)$ such that $Y=Y^{\prime}$ and $T=T^{\prime}$. Then $\rho(\mathscr{R})=\rho\left(\mathscr{R}^{\prime}\right)$.

Proof If $T=T^{\prime}$ then $\psi_{K}=\psi_{K}^{\prime}$. Hence, for every reactant complex $j \in \mathscr{C}$, it is the case that $\psi_{K j}=\psi_{K j}^{\prime}$ and both are nonzero. It follows that complex $j$ is in $\rho\left(\mathscr{R}^{\prime}\right)$. Similarly, for every reactant complex $j \in \mathscr{C}^{\prime}, j$ is in $\rho(\mathscr{R})$.

\subsection{Adaptation of the Johnston et al. MILP algorithm to PL-RDK systems}

Using the JSC, M.D. Johnston and collaborators developed several MILP algorithms to find linearly conjugate networks with known MAK dynamics. In [8], Johnston et al. specified a MILP algorithm to find linear conjugates which are weakly reversible. In further papers $[11,14]$ they refined their approach to construct linearly conjugate systems which are not only weakly reversible but also have desirable deficiency properties: minimal deficiency and satisfying the Deficiency One Theorem conditions. Since 
Deficiency Theory for PL-RDK systems is little developed (s. [21] for a detailed discussion), we focus only on extending the first algorithm for constructing weakly reversible linear conjugate systems.

\subsubsection{Key components of the Johnston et al. algorithm for MAK systems}

The algorithm considers two mass action systems; the original system $\mathscr{N}=$ $(\mathscr{S}, \mathscr{C}, \mathscr{R})$ and the target system $\mathscr{N}^{\prime}=\left(\mathscr{S}^{\prime}, \mathscr{C}^{\prime}, \mathscr{R}^{\prime}\right)$. The objective of the algorithm is the determination of the network structure of the target system and its corresponding rate constants which satisfies the weak reversibility and linear conjugacy conditions. The algorithm assumes that the two networks share the same set of species and complexes (i.e. $\mathscr{S}=\mathscr{S}^{\prime}$ and $\mathscr{C}=\mathscr{C}^{\prime}$ ), an assumption which implies that both the original and the target systems have the same molecularity matrix $Y$ and coefficient map $\psi(x)$. Also, $\mathscr{R}$ and $\mathscr{K}$ are known while $\mathscr{R}^{\prime}$ and $\mathscr{K}^{\prime}$ are to be determined.

The algorithm requires the following values to be determined before the implementation.

- The molecularity matrix $Y \in \mathbb{R}_{\geqslant 0}^{m \times n}$

- The reaction structure $R$ and the rate constant $K$ of the original system or the matrix $M=Y \cdot A_{k}$, where $A_{k}$ is the Kirchhoff matrix

- The sufficiently small parameter $\epsilon>0$ which is used to establish strict inequalities as permissible nonstrict inequalities

- The parameter $u_{i j}>0, i, j=1, \ldots, m, i \neq j$

\subsubsection{Discussion of the MILP algorithm for PL-RDK}

The MILP model which searches for a dense weakly reversible linearly conjugate network is given as follows. The parameters and variables used are found in Tables 1 and 2.

The objective is to find a dense realization of the given network that seeks to maximize the number of present reactions denoted by $\delta$.

$$
\operatorname{minimize} \sum_{i, j=1}^{m}-\delta_{i j}
$$

There are four sets of constraints in the MILP model which incorporate the desired structure of the network, weak reversibility requirement and linear conjugacy condition.

$$
\text { (WR) }\left\{\begin{array}{l}
\sum_{i=1, i \neq j}^{m}\left[\tilde{A}_{k}\right]_{i j}=\sum_{i=1, i \neq j}^{m}\left[\tilde{A}_{k}\right]_{j i}, \quad j=1, \ldots, m \\
{\left[\tilde{A}_{k}\right]_{i j} \geqslant 0, \quad \text { for } i=1, \ldots, m, i \neq j}
\end{array}\right.
$$


Table 1 List of variables used in the MILP

\begin{tabular}{ll}
\hline Notation & Description \\
\hline$\delta_{i j}, i, j=1, \ldots, m$ & $\begin{array}{l}\text { Binary variable that keeps track of the presence of the reaction in the target } \\
\text { network }\end{array}$ \\
{$\left[A_{b}\right]_{i j}, i, j=1,2, \ldots, m$} & $\begin{array}{l}\text { Kinetic matrix with the same structure as the target network } \\
{\left[\tilde{A}_{k}\right]_{i j}, i, j=1,2, \ldots, m}\end{array}$ \\
$\begin{array}{l}\text { A dummy matrix used to linearize the constraint that guarantees weak } \\
\text { reversibility }\end{array}$ \\
$\begin{array}{l}\text { A vector which is an element of } \mathbb{R}_{>0}^{n} \\
\text { A diagonal matrix diag }(c) \text { with vector } c \in \mathbb{R}_{>0}^{n}\end{array}$ \\
\hline
\end{tabular}

Table 2 List of parameters defined in the MILP

\begin{tabular}{ll}
\hline Notation & Description \\
\hline$Y$ & Complex matrix of the original network \\
$M$ & Product of $Y$ and the kinetic matrix $A_{k}$ of the original network \\
$u_{i j}, i, j=1, \ldots, m$ & A positive-valued parameter \\
$\varepsilon$ & A sufficiently small parameter with value between 0 to 1 \\
\hline
\end{tabular}

$$
\begin{gathered}
\text { (LC) }\left\{\begin{array}{l}
Y \cdot A_{b}=T^{-1} \cdot M, T=\operatorname{diag}\{c\} \\
\sum_{i=1, i \neq j}^{m}\left[A_{b}\right]_{i j}=0, \quad j=1, \ldots, m \\
{\left[A_{b}\right]_{i j} \geqslant 0, \quad \text { for } i=1, \ldots, m, i \neq j} \\
{\left[A_{b}\right]_{i i}<0, \quad \text { for } i=1, \ldots, m} \\
\epsilon \leqslant c_{i} \leqslant \frac{1}{\epsilon}, \quad \text { for } i=1, \ldots, n
\end{array}\right. \\
(\mathbf{W R}-\mathbf{S})\left\{\begin{array}{l}
0 \leqslant-\left[\tilde{A}_{k}\right]_{i j}+u_{i j} \cdot \delta_{i j}, \quad i, j=1, \ldots, m, i \neq j \\
0 \leqslant\left[\tilde{A}_{k}\right]_{i j}-\epsilon \cdot \delta_{i j}, \quad i, j=1, \ldots, m, i \neq j
\end{array}\right. \\
(\mathrm{LC}-\mathbf{S})\left\{\begin{array}{l}
0 \leqslant-\left[A_{b}\right]_{i j}+u_{i j} \cdot \delta_{i j}, \quad i, j=1, \ldots, m, i \neq j \\
0 \leqslant\left[A_{b}\right]_{i j}-\epsilon \cdot \delta_{i j}, \quad i, j=1, \ldots, m, i \neq j \\
\delta_{i j} \in\{0,1\} \quad \text { for } i, j=1, \ldots, m, i \neq j
\end{array}\right.
\end{gathered}
$$

Constraint set (4) checks weak reversibility of the chemical reaction network. Constraint set (5) imposes the linear conjugacy requirements. Constraint sets (6) and (7) are additional sets of conditions to ensure that the target chemical reaction network has the correct structure. A detailed discussion of the MILP formulation can be found in [13].

An alternative form of the objection function (i.e. $\sum_{i, j=1}^{m} \delta_{i j}$ ) over the same set of constraints can be used to determine the sparse realization of the network.

In a MAK system, dense/sparse weakly reversible linearly conjugate network can be determined by implementing the MILP algorithm and solving for $A_{b}$ and the vector $c$. The kinetics matrix corresponding to the target network is calculated by applying (2). 
For a PL-RDK system, the same algorithm can be employed only that the computation of the target Kirchhoff matrix is given as

$$
A_{k}^{\prime}=A_{b} \cdot D
$$

where

$$
D=\operatorname{diag}\{e\} \text { and } e_{j}= \begin{cases}c^{F \cdot j}, & \text { if complex } j \text { is a reactant of some reaction } k \\ 1, & \text { otherwise }\end{cases}
$$

It is important to note that the algorithm does not guarantee that the target network will have the same number of complexes as the original. In order to account for this discrepancy, a modification of the definition of a CRN is adopted, that is, a complex is allowed to react trivially to itself. This additional condition does not affect the determination of properties such as the deficiency and the weak reversibility of a network.

To summarize, the main difference between the MILP algorithm, for MAK and our extension lies in the consideration of the entries of the kinetic order matrix (or equivalently of the $T$ matrix) in the calculation of $A_{k}^{\prime}$. However, the $T$ matrix affects only the values of the rate constants in the factor $I_{k}^{\prime}$ of $A_{k}^{\prime}$, but not the entries in the incidence matrix factor $I_{a}^{\prime}$ of $A_{k}$.

\section{Results and discussion}

In this section, we present five examples of PL-RDK systems to illustrate the MILP implementation described in Sect. 4.2.2. The networks considered are taken from $[5,8,13,14]$. Two of the examples are nonbranching networks accounting for trivial and nontrivial linear conjugacy mappings. The third example considers a branching network. The last two examples are BST models.

The algorithm was implemented using GUSEK (GLPK Under Scite Extended Kit) [2], an open source integrated development environment which provides tools and facilities for solving LP and MILP problems.

Example 2 We consider the chemical reaction network $P$ defined by

$$
P:\left\{\begin{array}{l}
X_{1}+2 X_{2} \rightarrow X_{1} \\
2 X_{1}+X_{2} \rightarrow 3 X_{2} \\
X_{1}+3 X_{2} \rightarrow X_{1}+X_{2} \rightarrow 3 X_{1}+X_{2}
\end{array}\right.
$$

This network was considered in [13] as a MAK system in which a dense and sparse linearly conjugate weakly reversible networks were determined through the one-step MILP algorithm discussed in Sect. 4.2.2. Moreover, this network was also studied in $[12,20]$. Network $P$ has the corresponding molecularity matrix $Y$ and matrix $M$. 


$$
\begin{aligned}
Y & =\left[\begin{array}{lllllll}
1 & 1 & 2 & 0 & 1 & 1 & 3 \\
2 & 0 & 1 & 3 & 3 & 1 & 1
\end{array}\right] \\
M & =Y A_{k}=\left[\begin{array}{cccccccc}
0 & 0 & -2 & 0 & 0 & 2 & 0 \\
-3 & 0 & 2 & 0 & -2 & 0 & 0
\end{array}\right] .
\end{aligned}
$$

For the implementation of the algorithm, we endowed network $P$ with power-law kinetics to form a PL-RDK system. The system's transformed kinetic order matrix $T$ is

$$
T=\left[\begin{array}{cccc}
0.5 & 0.2 & 0.5 & 0.3 \\
1 & 1 & 1 & 0.2
\end{array}\right]
$$

The associated SFRF of the PL-RDK system $P$ is given by

$$
Y A_{k}^{\prime} \psi_{k}(X)=\left[\begin{array}{ccccccc}
0 & 0 & -2 & 0 & 0 & 2 & 0 \\
-3 & 0 & 2 & 0 & -2 & 0 & 0
\end{array}\right] \psi_{k}(X) \text {. }
$$

The algorithm described in [13] with the modification described in Sect. 4.2.2 is capable of determining either a dense or a sparse linearly conjugate weakly reversible network. A dense realization shown in Fig. 1 was determined by considering $\epsilon=\frac{2}{3}$ and $u_{i j}=20, j=1,2, \ldots, 7$ whenever $i \neq j$. The implementation yielded

$$
A_{b}=\left[\begin{array}{ccccccc}
-\frac{13}{3} & 0 & \frac{2}{3} & 0 & \frac{2}{3} & 0 & 0 \\
0 & 0 & 0 & 0 & 0 & 0 & 0 \\
0 & 0 & -2 & 0 & 0 & 2 & 0 \\
0 & 0 & 0 & 0 & 0 & 0 & 0 \\
\frac{2}{3} & 0 & \frac{2}{3} & 0 & -\frac{4}{3} & 0 & 0 \\
\frac{11}{3} & 0 & \frac{2}{3} & 0 & \frac{2}{3} & -2 & 0 \\
0 & 0 & 0 & 0 & 0 & 0 & 0
\end{array}\right]
$$

with both $c_{1}$ and $c_{2}$ equal to 1 . The values of the conjugacy constants imply that the linear transformation is an identity. Since $c$ is a trivial conjugacy vector, it follows that matrix $D$ is an identity. With this form of matrix $D$, the Kirchhoff matrix of the target network $P^{\prime}$ is $A_{k}^{\prime}=A_{b} \cdot D=A_{b}$. The network structure of network $P$ is given in Fig. 1.

Fig. 1 A dense weakly reversible realization that is linearly conjugate to $P$

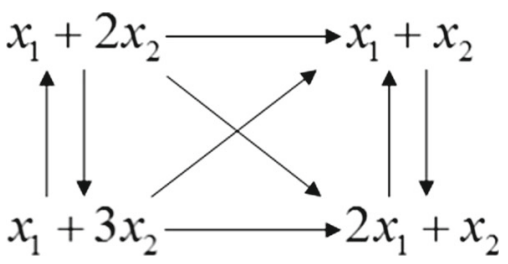


Fig. 2 A sparse weakly reversible realization that is linearly conjugate to $P$

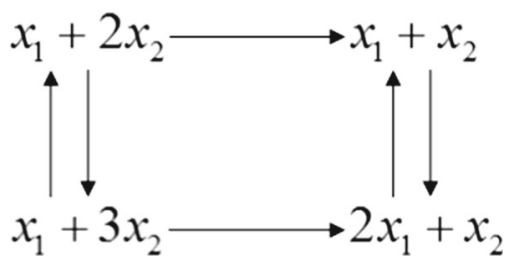

Example 3 In this example, we consider the same network $P$ as in Example 2. A sparse realization can also be generated by slightly modifying the objective function of the MILP as described in Sect. 4.2.2. Specifying the parameters $\epsilon=0.1$ and $u_{i j}=20, j=1,2, \ldots, 7$ for $i \neq j$, and considering the same $T$ matrix $T=T^{\prime}$ as in Example 2, the conjugacy constants $c_{1}=10$ and $c_{2}=5$ were obtained. Using (8), the corresponding Kirchhoff matrix of the target network $P$ was computed using (2).

$$
A_{k}^{\prime}=\left[\begin{array}{ccccccc}
-9.49 & 0 & 0 & 0 & 6.32 & 0 & 0 \\
0 & 0 & 0 & 0 & 0 & 0 & 0 \\
0 & 0 & -1.58 & 0 & 0 & 0.55 & 0 \\
0 & 0 & 0 & 0 & 0 & 0 & 0 \\
0 & 0 & 1.58 & 0 & -6.32 & 0 & 0 \\
9.49 & 0 & 0 & 0 & 0 & -0.55 & 0 \\
0 & 0 & 0 & 0 & 0 & 0 & 0
\end{array}\right]
$$

Network $P^{\prime \prime}$ is a weakly reversible linearly conjugate network with four complexes as presented in Fig. 2. This gives rise to the SFRF

$$
Y A_{k}^{\prime} \Psi_{k}(X)=\left\{\begin{array}{l}
-1.58 x_{1}^{0.2} x_{2}+0.55 x_{1}^{0.3} x_{2}^{0.2} \\
-9.49 x_{1}^{0.5} x_{2}+3.17 x_{1}^{0.2} x_{2}-6.32 x_{1}^{0.5} x_{2}
\end{array} .\right.
$$

Example 4 Consider a kinetic system

$$
\begin{aligned}
& \dot{x}_{1}=1-x_{1}^{2}-x_{1}+x_{2} x_{3} \\
& \dot{x}_{2}=2 x_{1}-2 x_{2} x_{3}-2 x_{2}^{2}+2 x_{3}^{2} \\
& \dot{x}_{3}=x_{1}-x_{2} x_{3}+x_{2}^{2}-x_{3}^{2}
\end{aligned}
$$

In [14], it was determined that the kinetic realization involves the following complexes: $C_{1}=x_{1}+x_{2}+x_{3}, C_{2}=x_{2}+x_{3}, C_{3}=x_{2}, C_{4}=x_{3}, C_{5}=2 x_{3}, C_{6}=x_{2}+2 x_{3}$, $C_{7}=2 x_{2}, C_{8}=2 x_{2}+x_{3}, C_{9}=0, C_{10}=x_{1}, C_{11}=2 x_{1}, C_{12}=x_{1}+x_{2}$, and 
$C_{13}=x_{1}+x_{3}$. The induced network structure is shown in Fig. 3. This gives rise to

$$
\begin{aligned}
& Y=\left[\begin{array}{ccccccccccccc}
1 & 0 & 0 & 0 & 0 & 0 & 0 & 0 & 0 & 1 & 2 & 1 & 1 \\
1 & 1 & 1 & 0 & 0 & 1 & 2 & 2 & 0 & 0 & 0 & 1 & 0 \\
1 & 1 & 0 & 1 & 2 & 2 & 0 & 1 & 0 & 0 & 0 & 0 & 1
\end{array}\right] \\
& \text { and } M=\left[\begin{array}{ccccccccccccc}
0 & 1 & 0 & 0 & 0 & 0 & 0 & 0 & 1 & -1 & -1 & 0 & 0 \\
0 & -2 & 0 & 0 & 2 & 0 & -2 & 0 & 0 & 2 & 0 & 0 & 0 \\
0 & -1 & 0 & 0 & -1 & 0 & 1 & 0 & 0 & 1 & 0 & 0 & 0
\end{array}\right] \text {. }
\end{aligned}
$$

The pairing of this network with the transformed kinetic order matrix

$$
T=\left[\begin{array}{cccccc}
0 & 0 & 0 & 0 & 0.2 & 0.3 \\
0.5 & 0 & -1 & 0 & 0 & 0 \\
1 & 2 & 0 & 0 & 0 & 0
\end{array}\right]
$$

yields the SFRF given below.

$$
\begin{aligned}
& \dot{x}_{1}^{\prime}=x_{2}^{0.5} x_{3}+1-x_{1}^{0.2}-x_{1}^{0.3} \\
& \dot{x}_{2}^{\prime}=-2 x_{2}^{0.5} x_{3}+2 x_{3}^{2}-2 x_{2}^{-1}+2 x_{1}^{0.2} \\
& \dot{x}_{3}^{\prime}=-x_{2}^{0.5} x_{3}-x_{3}^{2}+x_{2}^{-1}+x_{1}^{0.2}
\end{aligned}
$$

Employing the algorithm with parameter values $\epsilon=\frac{1}{10}$ and $u_{i j}=20$ whenever $i \neq j$, a linearly conjugate sparse realization, as shown in Fig. 4, with conjugacy constants $c_{1}=c_{3}=5$ and $c_{2}=10$ were determined. Solving for $A_{b}$, we have

$$
A_{b}=\left[\begin{array}{ccccccccccccc}
0 & 0 & 0 & 0 & 0 & 0 & 0 & 0 & 0 & 0 & 0 & 0 & 0 \\
0 & -0.2 & 0 & 0 & 0 & 0 & 0 & 0 & 0 & 0.2 & 0 & 0 & 0 \\
0 & 0 & 0 & 0 & 0 & 0 & 0 & 0 & 0 & 0 & 0 & 0 & 0 \\
0 & 0 & 0 & 0 & 0 & 0 & 0 & 0 & 0 & 0 & 0 & 0 & 0 \\
0 & 0 & 0 & 0 & -0.1 & 0 & 0.1 & 0 & 0 & 0 & 0 & 0 & 0 \\
0 & 0 & 0 & 0 & 0 & 0 & 0 & 0 & 0 & 0 & 0 & 0 & 0 \\
0 & 0 & 0 & 0 & 0.1 & 0 & -0.1 & 0 & 0 & 0 & 0 & 0 & 0 \\
0 & 0 & 0 & 0 & 0 & 0 & 0 & 0 & 0 & 0 & 0 & 0 & 0 \\
0 & 0 & 0 & 0 & 0 & 0 & 0 & 0 & -0.1 & 0 & 0.1 & 0 & 0 \\
0 & 0.2 & 0 & 0 & 0 & 0 & 0 & 0 & 0 & -0.2 & 0 & 0 & 0 \\
0 & 0 & 0 & 0 & 0 & 0 & 0 & 0 & 0.1 & 0 & -0.1 & 0 & 0 \\
0 & 0 & 0 & 0 & 0 & 0 & 0 & 0 & 0 & 0 & 0 & 0 & 0 \\
0 & 0 & 0 & 0 & 0 & 0 & 0 & 0 & 0 & 0 & 0 & 0 & 0
\end{array}\right] .
$$




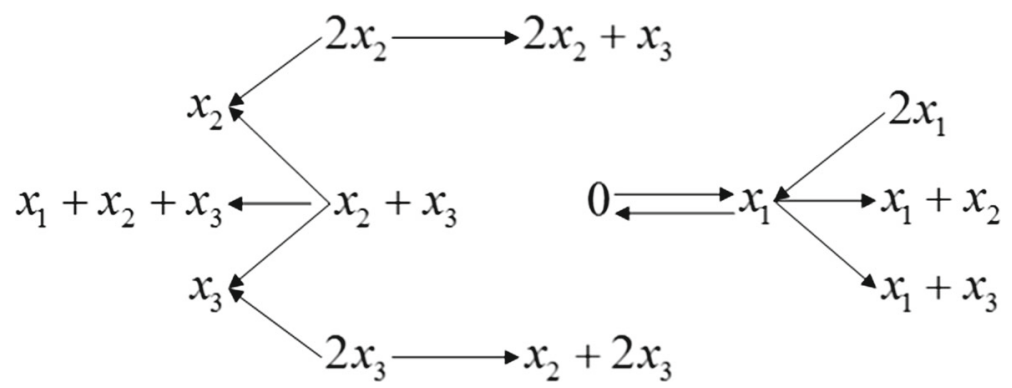

Fig. 3 A mass-action system representing the dynamics given of the kinetic system (9) with branching structure

Fig. 4 A sparse realization

capable of generating the

kinetics of the kinetic system (9)
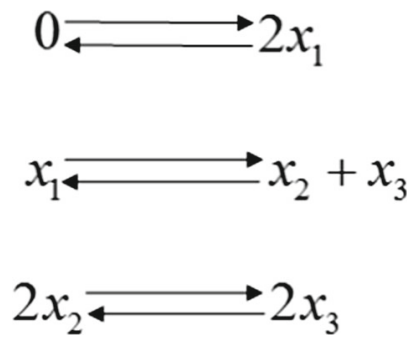

Using equation (8), the structure of the target system is determined and is embedded by

$$
A_{k}^{\prime}=\left[\begin{array}{ccccccccccccc}
0 & 0 & 0 & 0 & 0 & 0 & 0 & 0 & 0 & 0 & 0 & 0 & 0 \\
0 & -3.1623 & 0 & 0 & 0 & 0 & 0 & 0 & 0 & 0.276 & 0 & 0 & 0 \\
0 & 0 & 0 & 0 & 0 & 0 & 0 & 0 & 0 & 0 & 0 & 0 & 0 \\
0 & 0 & 0 & 0 & 0 & 0 & 0 & 0 & 0 & 0 & 0 & 0 & 0 \\
0 & 0 & 0 & 0 & 0 & 0 & 0 & 0 & 0 & 0 & 0 & 0 & 0 \\
0 & 0 & 0 & 0 & -2.5 & 0 & 0.01 & 0 & 0 & 0 & 0 & 0 & 0 \\
0 & 0 & 0 & 0 & 0 & 0 & 0 & 0 & 0 & 0 & 0 & 0 & 0 \\
0 & 0 & 0 & 0 & 2.5 & 0 & -0.01 & 0 & 0 & 0 & 0 & 0 & 0 \\
0 & 0 & 0 & 0 & 0 & 0 & 0 & 0 & -0.1 & 0 & 0.162 & 0 & 0 \\
0 & 3.1623 & 0 & 0 & 0 & 0 & 0 & 0 & 0 & -0.276 & 0 & 0 & 0 \\
0 & 0 & 0 & 0 & 0 & 0 & 0 & 0 & 0.1 & 0 & -0.162 & 0 & 0 \\
0 & 0 & 0 & 0 & 0 & 0 & 0 & 0 & 0 & 0 & 0 & 0 & 0 \\
0 & 0 & 0 & 0 & 0 & 0 & 0 & 0 & 0 & 0 & 0 & 0 & 0
\end{array}\right] .
$$

The resulting SFRF for the target network is

$$
\begin{aligned}
& \dot{x}_{1}^{\prime}=3.1623 x_{2}^{0.5} x_{3}+0.2-0.276 x_{1}^{0.2}-0.324 x_{1}^{0.3} \\
& \dot{x}_{2}^{\prime}=-3.1623 x_{2}^{0.5} x_{3}+5 x_{3}^{2}-0.02 x_{2}^{-1}+0.276 x_{1}^{0.2} \\
& \dot{x}_{3}^{\prime}=-3.1623 x_{2}^{0.5} x_{3}-5 x_{3}^{2}+0.02 x_{2}^{-1}+0.276 x_{1}^{0.2} .
\end{aligned}
$$

For the background on Biochemical Systems Theory (BST) and the CRN representations (total and embedded) of BST models needed for the next two examples, the reader is referred to the discussion in [1]. 
Example 5 Ray et al. [17] constructed a BST model describing the biochemical network of macrophages which are involved in host-pathogen interactions. The total representation is comprised of 12 species and 27 complexes in 21 reactions which are described below.

\begin{tabular}{ll}
\hline$R_{1}: X_{10}+X_{6} \rightarrow X_{10}+X_{6}+X_{1}$ & $R_{11}: X_{4}+X_{5} \rightarrow X_{4}+X_{5}+X_{6}$ \\
$R_{2}: X_{1} \rightarrow 0$ & $R_{12}: X_{6} \rightarrow 0$ \\
$R_{3}: X_{11}+X_{6} \rightarrow X_{11}+X_{6}+X_{2}$ & $R_{13}: X_{12}+X_{9} \rightarrow X_{12}+X_{9}+X_{7}$ \\
$R_{4}: X_{2} \rightarrow 0$ & $R_{14}: X_{7}+X_{8} \rightarrow X_{8}$ \\
$R_{5}: X_{1}+X_{2}+X_{7} \rightarrow X_{1}+X_{2}+X_{7}+X_{3}$ & $R_{15}: X_{9} \rightarrow X_{9}+X_{8}$ \\
$R_{6}: X_{3} \rightarrow 0$ & $R_{16}: X_{7}+X_{8} \rightarrow X_{7}$ \\
$R_{7}: X_{3} \rightarrow X_{3}+X_{4}$ & $R_{17}: 0 \rightarrow X_{9}$ \\
$R_{8}: X_{4} \rightarrow 0$ & $R_{18}: X_{6}+X_{7}+X_{9} \rightarrow X_{6}+X_{7}$ \\
$R_{9}: X_{4} \rightarrow X_{4}+X_{5}$ & $R_{19}: 0 \rightarrow X_{10}$ \\
$R_{10}: X_{4}+X_{5} \rightarrow X_{4}$ & $R_{20}: 0 \rightarrow X_{11}$ \\
& $R_{21}: 0 \rightarrow X_{12}$ \\
\hline
\end{tabular}

Here, $k_{1}=k_{2}=1.73, k_{3}=k_{4}=8.32, k_{5}=k_{6}=0.173, k_{7}=k_{8}=0.069$, $k_{9}=5.55, k_{10}=k_{11}=k_{12}=2.77, k_{13}=k_{14}=32.2, k_{15}=k_{16}=40, k_{17}=k_{18}=$ $k_{19}=k_{20}=k_{21}=36.7$. This network was endowed with the $T$ matrix

$$
T=\left[\begin{array}{cccccccccccccc}
1 & 0 & 0 & 0 & 0 & 0 & 0 & 0 & 0 & 0 & 1.19 & 0 & 0 & 0 \\
0 & 1 & 0 & 0 & 0 & 0 & 0 & 0 & 0 & 0 & 0.48 & 0 & 0 & 0 \\
0 & 0 & 1 & 0 & 0 & 0 & 0 & 0 & 0 & 0 & 0 & 0 & 0 & 0 \\
0 & 0 & 0 & 1 & 0 & 0 & 0 & 0 & 0 & 0 & 0 & 0 & 0 & 0 \\
0 & 0 & 0 & 0 & 1 & 0 & 0 & 0 & 0 & 0 & 0 & 0 & 0 & 0 \\
0 & 0 & 0 & 0 & 0 & 1 & 0 & 0 & -0.5 & -0.5 & 0 & 0 & 0 & -0.5 \\
0 & 0 & 0 & 0 & 0 & 0 & 0 & 0 & 0 & 0 & -0.177 & 1 & 0 & 0.5 \\
0 & 0 & 0 & 0 & 0 & 0 & 0 & 0 & 0 & 0 & 0 & 1 & 0 & 0 \\
0 & 0 & 0 & 0 & 0 & 0 & -0.645 & 0 & 0 & 0 & 0 & 0 & 0.5 & 1 \\
0 & 0 & 0 & 0 & 0 & 0 & 0 & 0 & 1 & 0 & 0 & 0 & 0 & 0 \\
0 & 0 & 0 & 0 & 0 & 0 & 0 & 0 & 0 & 1 & 0 & 0 & 0 & 0 \\
0 & 0 & 0 & 0 & 0 & 0 & 0 & 0 & 0 & 0 & 0 & 0 & 1 & 0
\end{array}\right]
$$

Implementation of the algorithm on the described network yielded no feasible solution. To allow for the generation of a sparse realization, the algorithm was relaxed by the removal of the constraints on weak reversibility. The nonweakly reversible linearly conjugate target network obtained by setting $\epsilon$ to 0.1 and $u_{i j}$ to 20 whenever $i \neq j$, described below has 18 reactions, 15 of which are also contained in the original network. The associated kinetic rates are $k_{1}{ }^{\prime}=0.5471, k_{2}{ }^{\prime}=8.32, k_{3}{ }^{\prime}=2.631$, $k_{4}{ }^{\prime}=8.32, k_{5}{ }^{\prime}=5.3788, k_{6}{ }^{\prime}=0.069, k_{7}{ }^{\prime}=0.383, k_{8}{ }^{\prime}=2.77, k_{9}{ }^{\prime}=2.77$, $k_{10}{ }^{\prime}=2.77, k_{11}{ }^{\prime}=126.49, k_{12}{ }^{\prime}=0.9039, k_{13}{ }^{\prime}=32.928, k_{14}{ }^{\prime}=k_{40}{ }^{\prime}=k_{15}{ }^{\prime}=3.67$, $k_{16}{ }^{\prime}=322, k_{17^{\prime}}=0.104, k_{18}^{\prime}=2.77$.

Since it is the embedded representation of a BST system, which is dynamically equivalent to it, we derive this for the original and target system and compare them in the following. Note that since the embedded systems are derived from the total systems by setting the independent variables constant, the linear conjugacy relationship is maintained. 


\begin{tabular}{ll}
$R_{1}^{\prime}: X_{10}+X_{6} \rightarrow X_{10}+X_{6}+X_{1}$ & $R_{10^{\prime}}: X_{6} \rightarrow 0$ \\
$R_{2}^{\prime}: X_{1} \rightarrow 0$ & $R_{11^{\prime}}: X_{12}+X_{9} \rightarrow X_{12}+X_{9}+X_{7}$ \\
$R_{3}^{\prime}: X_{11}+X_{6} \rightarrow X_{11}+X_{6}+X_{2}$ & $R_{12^{\prime}}: X_{9} \rightarrow X_{9}+X_{8}$ \\
$R_{4}^{\prime}: X_{2} \rightarrow 0$ & $R_{13^{\prime}}: X_{6}+X_{7}+X_{9} \rightarrow X_{6}+X_{7}$ \\
$R_{5}^{\prime}: X_{1}+X_{2}+X_{7} \rightarrow X_{1}+X_{2}+X_{7}+X_{3}$ & $R_{14^{\prime}}: 0 \rightarrow X_{10}$ \\
$R_{6}^{\prime}: X_{4} \rightarrow 0$ & $R_{15^{\prime}}: 0 \rightarrow X_{11}$ \\
$R_{7^{\prime}}: X_{4} \rightarrow X_{4}+X_{5}$ & $R_{16^{\prime}}: 0 \rightarrow X_{9}+X_{12}$ \\
$R_{8}^{\prime}: X_{4}+X_{5} \rightarrow X_{4}$ & $R_{17^{\prime}}: X_{7}+X_{8} \rightarrow 0$ \\
$R_{9}^{\prime}: X_{4}+X_{5} \rightarrow X_{4}+X_{5}+X_{6}$ & $R_{18^{\prime}}: X_{3} \rightarrow X_{4}$ \\
\hline
\end{tabular}

The embedded representation of the original system is:

\begin{tabular}{ll}
\hline$R_{E 1}: X_{6} \rightarrow X_{6}+X_{1}$ & $R_{E 10}: X_{4}+X_{5} \rightarrow X_{4}$ \\
$R_{E 2}: X_{1} \rightarrow 0$ & $R_{E 11}: X_{4}+X_{5} \rightarrow X_{4}+X_{5}+X_{6}$ \\
$R_{E 3}: X_{6} \rightarrow X_{6}+X_{2}$ & $R_{E 12}: X_{6} \rightarrow 0$ \\
$R_{E 4}: X_{2} \rightarrow 0$ & $R_{E 13}: X_{9} \rightarrow X_{9}+X_{7}$ \\
$R_{E 5}: X_{1}+X_{2}+X_{7} \rightarrow X_{1}+X_{2}+X_{7}+X_{3}$ & $R_{E 14}: X_{7}+X_{8} \rightarrow X_{8}$ \\
$R_{E 6}: X_{3} \rightarrow 0$ & $R_{E 15}: X_{9} \rightarrow X_{9}+X_{8}$ \\
$R_{E 7}: X_{3} \rightarrow X_{3}+X_{4}$ & $R_{E 16}: X_{7}+X_{8} \rightarrow X_{7}$ \\
$R_{E 8}: X_{4} \rightarrow 0$ & $R_{E 17}: 0 \rightarrow X_{9}$ \\
$R_{E 9}: X_{4} \rightarrow X_{4}+X_{5}$ & $R_{E 18}: X_{6}+X_{7}+X_{9} \rightarrow X_{6}+X_{7}$ \\
\hline
\end{tabular}

The embedded representation of the target system is:

\begin{tabular}{|c|c|}
\hline $\begin{array}{l}R_{E 1^{\prime}}: X_{6} \rightarrow X_{6}+X_{1} \\
R_{E 2^{\prime}}: X_{1} \rightarrow 0 \\
R_{E 3^{\prime}}: X_{6} \rightarrow X_{6}+X_{2} \\
R_{E 4^{\prime}}: X_{2} \rightarrow 0 \\
R_{E 5^{\prime}}: X_{1}+X_{2}+X_{7} \rightarrow X_{1}+X_{2}+X_{7}+X_{3} \\
R_{E 6^{\prime}}: X_{4} \rightarrow 0 \\
R_{E 7^{\prime}}: X_{4} \rightarrow X_{4}+X_{5} \\
R_{E 8^{\prime}}: X_{4}+X_{5} \rightarrow X_{4} \\
\end{array}$ & $\begin{array}{l}R_{E 9^{\prime}}: X_{4}+X_{5} \rightarrow X_{4}+X_{5}+X_{6} \\
R_{E 10^{\prime}}: X_{6} \rightarrow 0 \\
R_{E 11^{\prime}}: X_{9} \rightarrow X_{9}+X_{7} \\
R_{E 12^{\prime}}: X_{9} \rightarrow X_{9}+X_{8} \\
R_{E 13^{\prime}}: X_{6}+X_{7}+X_{9} \rightarrow X_{6}+X_{7} \\
R_{E 14^{\prime}}: 0 \rightarrow X_{9} \\
R_{E 15^{\prime}}: X_{7}+X_{8} \rightarrow 0 \\
R_{E 16^{\prime}}: X_{3} \rightarrow X_{4}\end{array}$ \\
\hline
\end{tabular}

Example 6 A summary of the characteristics of the embedded systems in Example 5 is provided in Table 3. Consider the anaerobic fermentation pathway from glucose to ethanol, glycerol, and polysaccharide in the yeast Saccharomyces cerevisiae given in [5], and originally described by Galazzo and Bailey [8]. The total representation of the S-system model is given below.

The algorithm, in the absence of the weak reversibility contraints, was implemented on the network embedded with kinetic order parameters, as shown in its $T$ matrix. Moreover, the kinetic rates for the 22 reactions are set as $k_{1}=0.5, k_{2}=1.2, k_{3}=$ $0.4, k_{4}=2.3, k_{5}=1.5, k_{6}=2, k_{7}=0.7, k_{8}=1.3, k_{9}=3, k_{10}=2.5, k_{11}=$ 
Table 3 Summary of the details of the embedded systems from Example 5

\begin{tabular}{llll}
\hline Embedded system & No. of complexes/ no. of reactions & Reactions in O not in T & Reactions in T not in O \\
\hline Original = O & $21 / 8$ & $R_{E 6}, R_{E 7}$ & \\
& & $R_{E 14}, R_{E 16}$ & $R_{E 15}^{\prime}, R_{E 16}^{\prime}$ \\
\hline
\end{tabular}

\begin{tabular}{ll}
\hline$R_{1}: X_{2}+X_{6} \rightarrow X_{1}+X_{2}$ & $R_{12}: X_{3}+X_{4}+X_{5}+X_{10} \rightarrow 2 X_{5}$ \\
$R_{2}: X_{1}+X_{5}+X_{7} \rightarrow X_{2}+X_{5}+X_{7}$ & $+X_{3}+X_{4}+X_{10}$ \\
$R_{3}: X_{1}+2 X_{5}+X_{7} \rightarrow X_{1}+X_{5}+X_{7}$ & $R_{13}: 2 X_{5}+X_{13} \rightarrow X_{5}+X_{13}$ \\
$R_{4}: X_{2}+X_{5}+X_{8} \rightarrow X_{3}+X_{5}+X_{8}$ & $R_{14}: 0 \rightarrow X_{6}$ \\
$R_{5}: 2 X_{2}+X_{5}+X_{8} \rightarrow X_{2}+X_{5}+X_{8}$ & $R_{15}: 0 \rightarrow X_{7}$ \\
$R_{6}: X_{2}+X_{5}+X_{11} \rightarrow X_{5}+X_{11}$ & $R_{16}: 0 \rightarrow X_{8}$ \\
$R_{7}: X_{2}+X_{5}+X_{11} \rightarrow X_{2}+X_{11}$ & $R_{17}: 0 \rightarrow X_{9}$ \\
$R_{8}: X_{3}+X_{5}+X_{9}+X_{14} \rightarrow X_{4}+X_{5}+X_{9}+X_{14}$ & $R_{18}: 0 \rightarrow X_{10}: 0 \rightarrow X_{11}$ \\
$R_{9}: X_{3}+X_{5}+X_{9}+X_{14} \rightarrow X_{3}+2 X_{5}+X_{9}+X_{14}$ & $R_{20}: 0 \rightarrow X_{12}$ \\
$R_{10}: X_{3}+X_{4}+X_{5}+X_{12} \rightarrow X_{4}+X_{5}+X_{12}$ & $R_{21}: 0 \rightarrow X_{13}$ \\
$R_{11}: X_{3}+X_{4}+X_{5}+X_{10} \rightarrow X_{3}+X_{5}+X_{10}$ & $R_{22}: 0 \rightarrow X_{14}$ \\
\hline
\end{tabular}

$1.8, k_{12}=2.4, k_{13}=3.1, k_{14}=1, k_{15}=1, k_{16}=1, k_{17}=1, k_{18}=1, k_{19}=$ $1, k_{20}=1, k_{21}=1, k_{22}=1$.

$c T=\left[\begin{array}{ccccccccccc}0 & 0.7464 & 0.7464 & 0 & 0 & 0 & 0 & 0 & 0 & 0 & 0 \\ -0.2344 & 0 & 0 & 0.7318 & 0.7318 & 8.6107 & 0 & 0 & 0 & 0 & 0 \\ 0 & 0 & 0 & 0 & 0 & 0 & 0.6159 & 0.05 & 0.05 & 0 & 0 \\ 0 & 0 & 0 & 0 & 0 & 0 & 0 & 0.533 & 0.533 & 0 & 0 \\ 0 & 0.0243 & 0.0243 & -0.3941 & -0.3941 & 0 & 0.1308 & -0.0822 & -0.0822 & 1 & 0 \\ 1 & 0 & 0 & 0 & 0 & 0 & 0 & 0 & 0 & 0 & 0 \\ 0 & 1 & 1 & 0 & 0 & 0 & 0 & 0 & 0 & 0 & 0 \\ 0 & 0 & 0 & 1 & 1 & 0 & 0 & 0 & 0 & 0 & 0 \\ 0 & 0 & 0 & 0 & 0 & 0 & 1 & 0 & 0 & 0 & 0 \\ 0 & 0 & 0 & 0 & 0 & 0 & 0 & 0 & 1 & 0 & 0 \\ 0 & 0 & 0 & 0 & 0 & 1 & 0 & 0 & 0 & 0 & 0 \\ 0 & 0 & 0 & 0 & 0 & 0 & 0 & 1 & 0 & 0 & 0 \\ 0 & 0 & 0 & 0 & 0 & 0 & 0 & 0 & 0 & 1 & 0 \\ 0 & 0 & 0 & 0 & 0 & 0 & -0.6088 & 0 & 0 & 0 & 0\end{array}\right]$

With parameter values $\epsilon=0.04$ and $u_{i j}=20, i, j=1,2, \ldots, 31, i \neq j$, the target network is determined using the MILP algorithm. The target network has 21 reactions with reaction rates given below. Reactions 6 and 7 are replaced by $X_{2}+X_{5}+X_{11} \rightarrow$ $X_{11}$. 


\begin{tabular}{ll}
\hline$R_{1}^{\prime}: X_{2}+X_{6} \rightarrow X_{1}+X_{2}$ & $R_{11^{\prime}}: X_{3}+X_{4}+X_{5}+X_{10} \rightarrow 2 X_{5}$ \\
$R_{2}^{\prime}: X_{1}+X_{5}+X_{7} \rightarrow X_{2}+X_{5}+X_{7}$ & $+X_{3}+X_{4}+X_{10}$ \\
$R_{3}^{\prime}: X_{1}+2 X_{5}+X_{7} \rightarrow X_{1}+X_{5}+X_{7}$ & $R_{12}^{\prime}: 2 X_{5}+X_{13} \rightarrow X_{5}+X_{13}$ \\
$R_{4}^{\prime}: X_{2}+X_{5}+X_{8} \rightarrow X_{3}+X_{5}+X_{8}$ & $R_{13}^{\prime}: 0 \rightarrow X_{6}$ \\
$R_{5}^{\prime}: 2 X_{2}+X_{5}+X_{8} \rightarrow X_{2}+X_{5}+X_{8}$ & $R_{14}^{\prime}: 0 \rightarrow X_{7}$ \\
$R_{6}^{\prime}: X_{2}+X_{5}+X_{11} \rightarrow X_{11}$ & $R_{15}^{\prime}: 0 \rightarrow X_{8}$ \\
$R_{7}^{\prime}: X_{3}+X_{5}+X_{9}+X_{14} \rightarrow X_{4}+X_{5}+X_{9}+X_{14}$ & $E_{16^{\prime}}: 0 \rightarrow X_{9}: 0 \rightarrow X_{10}$ \\
$R_{8}^{\prime}: X_{3}+X_{5}+X_{9}+X_{14} \rightarrow X_{3}+2 X_{5}+X_{9}+X_{14}$ & $R_{18}^{\prime}: 0 \rightarrow X_{11}$ \\
$R_{9}^{\prime}: X_{3}+X_{4}+X_{5}+X_{12} \rightarrow X_{4}+X_{5}+X_{12}$ & $R_{19}^{\prime}: 0 \rightarrow X_{12}$ \\
$R_{10}:: X_{3}+X_{4}+X_{5}+X_{10} \rightarrow X_{3}+X_{5}+X_{10}$ & $R_{20}^{\prime}: 0 \rightarrow X_{13}$ \\
& $R_{21}^{\prime}: 0 \rightarrow X_{14}$ \\
\hline
\end{tabular}

Here, $k_{1}^{\prime}=0.276601, \quad k_{2}^{\prime}=16.3878, k_{3}^{\prime}=15.60743, \quad k_{4}^{\prime}=16.32552, \quad k_{5}^{\prime}=$ $30.42023, \quad k_{6}^{\prime}=1.11 \times 10^{1} 0, \quad k_{7}^{\prime}=2.10540, \quad k_{8}^{\prime}=13.88175, \quad k_{9}^{\prime}=$ $19.30991, k_{10}^{\prime}=13.90313, k_{11}^{\prime}=52.96431, \quad k_{12}^{\prime}=60, k_{13}^{\prime}=0.08, \quad k_{14}^{\prime}=$ $k_{15}^{\prime}=k_{16}^{\prime}=k_{17}^{\prime}=k_{18}^{\prime}=k_{19}^{\prime}=k_{20}^{\prime}=k_{21}^{\prime}=0.04$.

The embedded representation of the original system is:

\begin{tabular}{ll}
$R_{E 1}: X_{2} \rightarrow X_{1}+X_{2}$ & $R_{E 7}: X_{2}+X_{5} \rightarrow X_{2}$ \\
$R_{E 2}: X_{1}+X_{5} \rightarrow X_{2}+X_{5}$ & $R_{E 8}: X_{3}+X_{5} \rightarrow X_{4}+X_{5}$ \\
$R_{E 3}: X_{1}+2 X_{5} \rightarrow X_{1}+X_{5}$ & $R_{E 9}: X_{3}+X_{5} \rightarrow X_{3}+2 X_{5}$ \\
$R_{E 4}: X_{2}+X_{5} \rightarrow X_{3}+X_{5}$ & $R_{E 10}: X_{3}+X_{4}+X_{5} \rightarrow X_{4}+X_{5}$ \\
$R_{E 5}: 2 X_{2}+X_{5} \rightarrow X_{2}+X_{5}$ & $R_{E 11}: X_{3}+X_{4}+X_{5} \rightarrow X_{3}+X_{5}$ \\
$R_{E 6}: X_{2}+X_{5} \rightarrow X_{5}$ & $R_{E 12}: X_{3}+X_{4}+X_{5} \rightarrow 2 X_{5}+X_{3}+X_{4}$ \\
& $R_{E 13}: 2 X_{5} \rightarrow X_{5}$ \\
\hline
\end{tabular}

The embedded representation of the target system is:

\begin{tabular}{|c|c|}
\hline $\begin{array}{l}R_{E 1^{\prime}}: X_{2} \rightarrow X_{1}+X_{2} \\
R_{E 2^{\prime}}: X_{1}+X_{5} \rightarrow X_{2}+X_{5} \\
R_{E 3^{\prime}}: X_{1}+2 X_{5} \rightarrow X_{1}+X_{5} \\
R_{E 4^{\prime}}: X_{2}+X_{5} \rightarrow X_{3}+X_{5} \\
R_{E 5^{\prime}}: 2 X_{2}+X_{5} \rightarrow X_{2}+X_{5} \\
R_{E 6^{\prime}}: X_{2}+X_{5} \rightarrow 0\end{array}$ & $\begin{array}{l}R_{E 7^{\prime}}: X_{3}+X_{5} \rightarrow X_{4}+X_{5} \\
R_{E 8^{\prime}}: X_{3}+X_{5} \rightarrow X_{3}+2 X_{5} \\
R_{E 9^{\prime}}: X_{3}+X_{4}+X_{5} \rightarrow X_{4}+X_{5} \\
R_{E 10^{\prime}}: X_{3}+X_{4}+X_{5} \rightarrow X_{3}+X_{5} \\
R_{E 11^{\prime}}: X_{3}+X_{4}+X_{5} \rightarrow 2 X_{5}+X_{3}+X_{4} \\
R_{E 12^{\prime}}: 2 X_{5} \rightarrow X_{5}\end{array}$ \\
\hline
\end{tabular}

\section{Conclusion and outlook}

Table 4 summarizes the characteristics of the embedded systems in Example 6. In conclusion, we summarize our main results and outline some perspectives for further research based on them.

Table 4 Summary of the details of the embedded systems from Example 6

\begin{tabular}{llll}
\hline Embedded system & No. of complexes/ no. of reactions & Reactions in O not in T & Reactions in T not in O \\
\hline Original $=\mathrm{O}$ & $14 / 13$ & $R_{E 6}, R_{E 7}$ & $R_{E 6}^{\prime}$ \\
Target $=\mathrm{T}$ & $14 / 12$ & & ${ }^{\prime}$ \\
\hline
\end{tabular}


1. We used the $T$ matrix description of a PL-RDK system to show that a necessary condition for linear conjugacy between factor span surjective (PL-FSK) systems on nonbranching CRNs is the equality $T=T^{\prime}$ of the $T$ matrices of the two systems.

2. We extended the Johnston-Siegel Criterion for Linear Conjugacy from MAK systems to PL-RDK systems satisfying the additional assumption that their $T$ matrices are equal.

3. We showed that the MILP algorithms of Szederkenyi [19] and Johnston et al. [13] with just a few changes could be used to determine linear conjugates of a given PL-RDK system under the assumption that the $T$ matrix remains the same.

4. We provided several computational examples, including kinetic systems derived from 2 complex BST models, for the extended algorithms.

5. The analysis by Arceo et al [1] of 15 BST case studies showed that most of the chemical kinetic systems derived from them, especially the embedded ones, were in $\operatorname{PL}-\mathrm{NDK}(\mathscr{N})$, the set of non-PL-RDK systems in $\operatorname{PLK}(\mathscr{N})$. The concepts of linear conjugacy of course apply to such systems, and in fact, in Examples 5 and 6, we used the linear conjugacy of the total systems (which are both PL-RDK) to infer the linear conjugacy of the embedded systems (which are both PL-NDK). To address the predominant case of non-RDK biochemical systems, we are extending the methods presented here in a more general context in [3].

Acknowledgements Open access funding provided by Max Planck Society. The authors thank M. Feinberg for a clarification of the outflow reaction concept in CRNT and D. Talabis for contributing computations from his MS thesis to Examples 5 and 6.

\section{Compliance with ethical standards}

Conflict of interest The authors declare that they have no conflict of interest.

Open Access This article is distributed under the terms of the Creative Commons Attribution 4.0 International License (http://creativecommons.org/licenses/by/4.0/), which permits unrestricted use, distribution, and reproduction in any medium, provided you give appropriate credit to the original author(s) and the source, provide a link to the Creative Commons license, and indicate if changes were made.

\section{References}

1. C. Arceo, E. Jose, A. Lao, E. Mendoza, Reaction networks and kinetics of biochemical systems. Math. Biosci. 283, 13-29 (2017)

2. L. Bettoni, Gusek (glpk under scite extended kit). http://gusek.sourceforge.net (2010-2015)

3. M. Cortez, A. Nazareno, E. Mendoza, Linear conjugacy in rid kinetic systems (in preparation)

4. G. Craciun, C. Pantea, Identifiability of chemical reaction networks. J. Math. Chem. 44, 244 (2008)

5. R. Curto, A. Sorribas, M. Cascante, Comparative characterization of the fermentation pathway of saccharomyces cerevisiae using biochemical systems theory and metabolic control analysis: model definition and nomenclature. Math. Biosci. 130(1), 25-50 (1995)

6. G. Farkas, Kinetic lumping schemes. Chem. Eng. Sci. 54(17), 3909-3915 (1999)

7. A. Gabor, K. Hangos, J. Banga, G. Szederkenyi, Reaction network realizations of rational biochemical systems and their structural properties. J. Math. Chem. 53, 1657-1686 (2015)

8. J. Galazzo, J. Bailey, Fermentation pathway kinetics and metabolic flux control in suspended and immobilized saccharomyces cerevisiae. Enzym. Microb. Technol. 12(3), 162-172 (1990)

9. V. Hars, J. Toth, On the inverse problem of reaction kinetics. Coll. Math. Soc. J. Bolyai 30, 363 (1981)

10. M. Johnston, A computational approach to steady state correspondence of regular networks and generalized mass action systems. Bull. Math. Biol. 77, 1065-1100 (2015) 
11. M. Johnston, A linear programming approach to dynamical equivalence, linear conjugacy, and the deficiency one theorem. J. Math. Chem. 54, 1612-1631 (2016)

12. M. Johnston, D. Siegel, Linear conjugacy of chemical reaction networks. J. Math. Chem. 49, 1263-1282 (2011)

13. M. Johnston, D. Siegel, G. Szederkenyi, A linear programming approach to weak reversibilityand linear conjugacy of chemical reaction networks. J. Math. Chem. 50, 274-288 (2012)

14. M. Johnston, D. Siegel, G. Szederkenyi, Computing weakly reversible linearly conjugate networks with minimal deficiency. Math. Biosci. 241, 88-98 (2013)

15. S. Müller, G. Regensburger, Generalized mass action systems: Complex balancing equilibria and sign vectors of the stoichiometric and kinetic order subspaces. SIAM J. Appl. Math. 72(6), 1926-1947 (2012)

16. S. Müller, G. Regensburger, Generalized mass action systems and positive solutions of polynomial equations with real and symbolic exponents, ed. by V.P. Gerdt, W. Koepf, W.M. Seiler, E.H. Vorozhtsov. Proceedings of CASC 2014. Lecture Notes in Computer Science. pp. 302-323 (2014)

17. J. Ray, D. Kirschner, Requirement for multiple activation signals by anti-inflammatory feedback in macrophages. J. Theor. Biol. 241, 276-294 (2006)

18. G. Szederkenyi, Comment on identifiability of chemical reaction networks by G. Craciun and C. Pantea. J. Math. Chem. 45, 1172 (2009)

19. G. Szederkenyi, Computing sparse and dense realizations of reaction kinetic systems. J. Math. Chem. 47, 551-568 (2010)

20. G. Szederkenyi, K. Hangos, Z. Tuza, Finding weakly reversible realizations of chemical reaction networks using optimization. MATCH Commun. Math. Comput. Chem. 67, 193-212 (2012)

21. D. Talabis, C. Arceo, E. Mendoza, Positive equilibria of a class of power law kinetics (2017, submitted) 